\title{
Applying Loop-Flower Basis Functions to Analyze Electromagnetic Scattering Problems of PEC Scatterers
}

\author{
Gaobiao Xiao \\ Key Laboratory of Ministry of Education of Design and Electromagnetic Compatibility of High-Speed Electronic Systems, \\ Shanghai Jiao Tong University, Shanghai 200240, China \\ Correspondence should be addressed to Gaobiao Xiao; gaobiaoxiao@sjtu.edu.cn
}

Received 1 December 2013; Revised 5 February 2014; Accepted 7 February 2014; Published 17 March 2014

Academic Editor: Ladislau Matekovits

Copyright (C) 2014 Gaobiao Xiao. This is an open access article distributed under the Creative Commons Attribution License, which permits unrestricted use, distribution, and reproduction in any medium, provided the original work is properly cited.

This paper discusses the application of loop-flower basis functions for solving surface integral equations involved in electromagnetic scattering problems on perfectly electrically conducting surfaces. Flower-shaped basis functions are proposed to replace the conventional star basis functions. The flower basis functions are defined based on mesh nodes instead of surface triangles. It is shown that the loop-flower basis functions not only can be used to handle the electromagnetic scattering problems at very low frequencies, but also can be directly used to implement Calderon preconditioners for EFIEs.

\section{Introduction}

The Rao-Wilton-Glisson (RWG) basis functions are wellused low-order basis functions for solving surface integral equations (SIEs) [1], such as in solving the widely-used electric field integral equation (EFIE). However, solving EFIE with RWG basis functions usually suffers from two problems: low frequency breakdown and the ill-conditioning of the coefficient matrix in the corresponding discrete system. At low frequencies, the electric field generated by the solenoidal part of the electric current is largely overwhelmed by that generated by the nonsolenoidal part of the current. Solving EFIE with RWG basis functions cannot provide accurate solutions to the electric currents. This is known as the low frequency breakdown problem and can be overcome using several techniques such as performing a quasi-Helmholtz decomposition [2-5]: expanding the surface currents with loop-tree or loop-star basis functions so that the surface currents are approximately divided into a solenoidal part and a nonsolenoidal part. EFIEs can be correctly solved at very low frequencies with this technique. However, the interaction matrix of the discretized system associated with EFIE is still ill-conditioned, which is intrinsically caused by the spectral properties of the EFIE operator. Many preconditioning techniques are developed to improve the behavior of the EFIEs. The Calderon preconditioners have recently attracted much attention because of the ability in manipulating the spectrum properties of EFIEs [6]. The main difficulty in implementing the Calderon preconditioner is to find a well-conditioned Gram matrix to link the range and domain of the EFIE operator. The entries of the Gram matrix can be generally expressed by $\left\langle\widehat{\mathbf{n}} \times \mathbf{f}_{1}, \mathbf{f}_{2}\right\rangle$, where $\mathbf{f}_{1}$ and $\mathbf{f}_{2}$ are, respectively, the basis functions for the right and the left EFIE operators in the Calderon preconditioned EFIEs and $\widehat{\mathbf{n}}$ is the normal unit vector. In the case of PEC scatterers, $\mathbf{f}_{1}$ is used for the surface electric currents and $\mathbf{f}_{2}$ is for the equivalent surface magnetic currents. If we choose RWG bases for both $\mathbf{f}_{1}$ and $\mathbf{f}_{2}$, the resultant Gram matrix is singular because the divergence conforming RWGs and the curl conforming $\widehat{\mathbf{n}} \times$ RWGs are approximately orthogonal. It is necessary to select a set of basis functions other than RWGs for $\mathbf{f}_{2}$. The Buffa-Christiansen basis functions (BCs) $[7,8]$ meet the requirements of the choice. BCs are approximately orthogonal to the primary RWG functions. They are divergence conforming and quasi-curl-conforming, so that the corresponding Gram matrix is well-conditioned. However, if implemented directly without special treatment for the hyper singular term, the EFIE solver using $\mathrm{BC}$ basis functions and Calderon preconditioner (BC-CP) may fail at very low frequencies due to truncation errors [9], which motivates 
the usage of the dual loop-star basis functions $[9,10]$ that are created from $\mathrm{BC}$ basis functions.

It is well known that by Helmholtz decomposition, the electric currents can be divided into solenoidal currents, which are divergence free, and nonsolenoidal currents that are curl-free. As discussed in [2], the solenoidal surface currents $\mathbf{J}^{\text {sol }}$ can be expressed by a scalar function as $\mathbf{J}^{\text {sol }}=$ $\nabla_{t} \times(\widehat{\mathbf{n}} M(\mathbf{r}))$, where $M(\mathbf{r})$ is called solenoidal potential and $\nabla_{t}=\nabla-\hat{\mathbf{n}} \partial / \partial n$. Therefore, the construction of basis functions for the vector $\mathrm{J}^{\text {sol }}$ is basically changed to how to represent the scalar function $M(\mathbf{r})$ with scalar basis functions. It is possible to adopt the node-based piecewise linear functions with support on the triangles that have a vertex at the reference node of the mesh. The resultant vector basis functions are the loop basis functions. Similarly, the nonsolenoidal currents $\mathbf{J}^{\mathrm{irr}}$ can also be represented by a scalar potential as $\mathbf{J}^{\text {irr }}=\nabla_{t} \phi(\mathbf{r})$, which implies that they can be handled starting from representing the scalar potential with scalar basis functions. On the other hand, the divergence of $\mathbf{J}^{\text {irr }}$ is proportional to the surface charge, which may be denoted by $\nabla_{t} \cdot \mathbf{J}^{\mathrm{irr}}=-j \omega \rho(\mathbf{r})$. This linkage again states that although $\mathbf{J}^{\text {irr }}$ are vector fields, they can indeed be handled using scalar fields. Therefore, the construction of basis functions for the vector current $\mathbf{J}^{\text {irr }}$ can also be transformed to represent the scalar potential field with scalar basis functions, and using node-based basis functions is a possible choice for $\mathbf{J}^{\text {irr }}$ too. However, the support of the basis may need to be modified in order that the resultant vector basis functions for $\mathbf{J}^{\text {irr }}$ are divergence-conforming. One important concern here is the number of degrees of freedom. The number of the degrees of freedom for bases with support on triangles has been investigated extensively [11-14], such as the RWG bases. A safe choice is to use the loop basis functions for the solenoidal currents and find a set of basis functions for $\mathrm{J}^{\text {irr }}$, making the total number of degrees of freedom of these two set of bases equal to that of the RWG bases. Star basis functions that are defined based on triangles strictly meet this requirement and have been successfully used for remedying low frequency breakdown problems. However, meeting the requirement of the degrees of freedom of RWGs is not a necessary condition for the node-based basis functions. Basis functions with different support may have different numbers of degrees of freedom. Many well-used basis functions become successful just because they have introduced new features to eliminate unnecessary degrees of freedom. For example, on a closed surface with a mesh structure containing $N$ triangles, the RWG bases have reduced the degrees of freedom from $3 \mathrm{~N}$ to $1.5 \mathrm{~N}$ by introducing the divergence conforming property. Since node-based functions are different from RWG bases, it is not necessary to require that the total number of the degrees of freedom be equal to that of the RWG basis functions. In other words, it is surely possible to use nodebased basis functions for both the solenoidal currents and the nonsolenoidal currents. Even if the total number of degrees of freedom of the node-based basis functions is not equal to that of RWGs, they can still form a complete set of basis functions. Based on the observation, the node-based

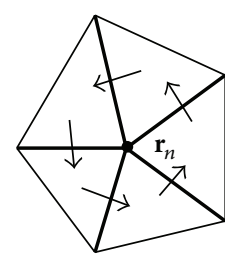

(a)

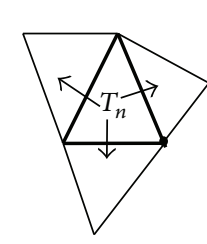

(b)

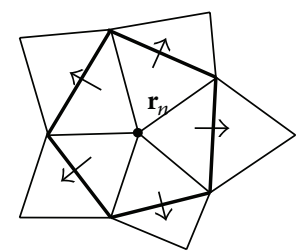

(c)
FIGURE 1: (a) Loop, (b) star, and (c) flower basis functions.

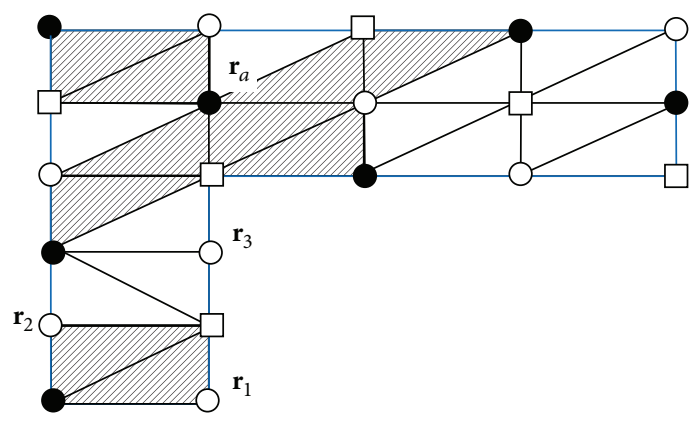

FIgURE 2: Flower basis functions near the boundary (blue line).

vector basis function for $\mathbf{J}^{\mathrm{irr}}$ is proposed, which is called flower basis function because the support of the basis looks like a flower. However, the application of loop-flower basis functions is confined to scatterers with closed-surfaces. This paper extends the application of loop-flower basis functions to scatterers with open surfaces. Detailed descriptions on implementing Calderon preconditioners using loop-flower basis functions are also provided, together with discussions on the properties of the flower basis functions.

\section{Definition of the Loop-Flower Basis Functions}

A loop basis function is defined with respect to the reference node $\mathbf{r}_{n}$, with support on all the triangles connecting to $\mathbf{r}_{n}$, as depicted in Figure 1(a). A reference circulation direction is assigned for each loop basis function. A loop basis function can be expressed by the aggregation of RWG basis functions. Denote the modified RWG basis as $\tilde{\mathbf{f}}_{n}^{\mathrm{RWG}}(\mathbf{r})=\mathbf{f}_{n}^{\mathrm{RWG}}(\mathbf{r}) / l_{n}$, where $l_{n}$ is the common edge length. The loop basis function is defined as follows:

$$
\mathbf{f}_{n}^{\mathrm{Loop}}(\mathbf{r})=\sum_{i=1}^{N_{n}} C_{n, i}^{\mathrm{Loop}} \widetilde{\mathbf{f}}_{n, i}^{\mathrm{RWG}}(\mathbf{r})
$$

where $N_{n}$ is the number of the surrounding triangles and $\tilde{\mathbf{f}}_{n, i}^{\mathrm{RWG}}(\mathbf{r})$ is the $i$ th modified RWG basis whose common edge connects to the node $\mathbf{r}_{n}$. We choose $C_{n, i}^{\text {Loop }}=1$ if $\tilde{\mathbf{f}}_{n, i}^{\mathrm{RWG}}(\mathbf{r})$ is in the same direction with the reference direction of the loop basis; otherwise, $C_{n, i}^{\text {Loop }}=-1$. In Figures 1 and 4 , common edges of RWGs are all plotted with bold lines.

A star basis function is defined with respect to the reference triangle $T_{n}$, as shown in Figure $1(\mathrm{~b})$, where its 


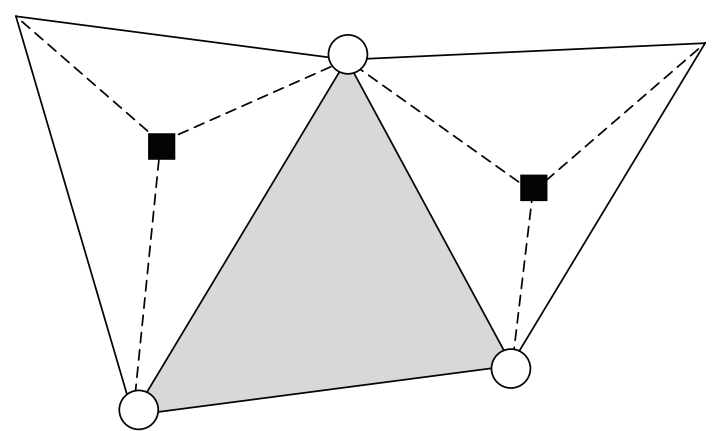

FIGURE 3: Modified mesh structure with 2 auxiliary nodes and 6 auxiliary edges.

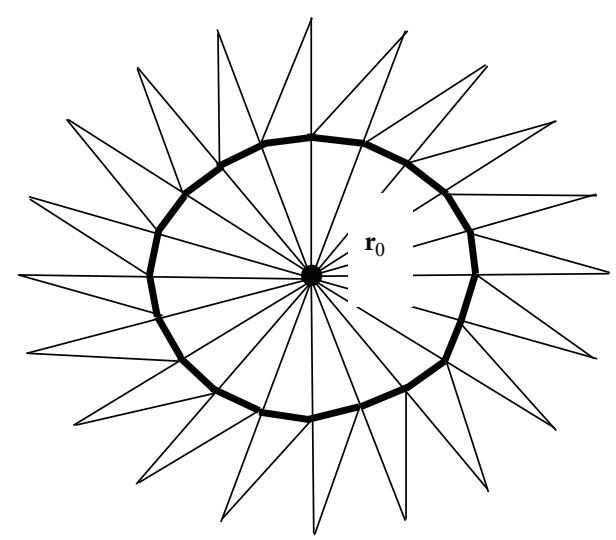

FIGURE 4: Irregularly-defined flower basis function.

reference direction is also depicted. A star basis function can be expressed by

$$
\mathbf{f}_{n}^{\mathrm{Star}}(\mathbf{r})=\sum_{i=1}^{N_{t}} C_{n, i}^{\mathrm{Star}} \tilde{\mathbf{f}}_{n, i}^{\mathrm{RWG}}(\mathbf{r}),
$$

where $N_{t}$ is the number of the RWG bases that contain the reference triangle. For star basis functions except those at the boundary of an open surface, we have $N_{t}=3$.

A flower basis function is defined with respect to the reference node $\mathbf{r}_{n}$, like a loop basis function, as shown in Figure 1(c). Its support covers all the RWG bases that take the reference node $\mathbf{r}_{n}$ as one free vertex. It can be explicitly expressed by

$$
\mathbf{f}_{n}^{\text {Flower }}(\mathbf{r})=\sum_{i=1}^{N_{n}} C_{n, i}^{\text {Flower }} \tilde{\mathbf{f}}_{n, i}^{\text {RWG }}(\mathbf{r}) .
$$

A reference direction is required too, as shown in Figure 1(c). Throughout this paper, it is assumed that the reference directions of the flower bases $\mathbf{f}_{n}^{\text {Flower }}(\mathbf{r})$ and those of the rotated loop bases $\widehat{\mathbf{n}} \times \mathbf{f}_{n}^{\text {Loop }}(\mathbf{r})$ are all pointing away from their reference nodes. The flower basis function and the loop basis function at a node contain the same number of RWGs.

The flower basis functions near the boundary of an open surface may become incomplete in shape. As shown in
Figure 2, the flower basis function at node $\mathbf{r}_{1}$ has only one qualified RWG, and that at $\mathbf{r}_{a}$ has only 4 qualified RWGs.

With the reference direction shown in Figure 1, a flower basis function can also be represented by star basis functions as follows:

$$
\mathbf{f}_{n}^{\text {Flower }}(\mathbf{r})=\sum_{i=1}^{N_{n}} \mathbf{f}_{n, i}^{\text {Star }}(\mathbf{r})
$$

where $\mathbf{f}_{n, i}^{\mathrm{Star}}(\mathbf{r})$ is the $i$ th star basis surrounding the node $\mathbf{r}_{n}$.

It is straight forward to check that

$$
\begin{array}{cc}
\nabla_{t} \cdot \mathbf{f}_{n}^{\text {Loop }}(\mathbf{r})=0, & \nabla_{t} \times \mathbf{f}_{n}^{\text {Loop }}(\mathbf{r}) \neq 0, \\
\nabla_{t} \times \mathbf{f}_{n}^{\text {Flower }}(\mathbf{r}) \approx 0, & \nabla_{t} \cdot \mathbf{f}_{n}^{\text {Flower }}(\mathbf{r}) \neq 0 .
\end{array}
$$

Therefore, loop-flower basis functions are suitable for performing quasi-Helmholtz decomposition of surface currents.

\section{Number of DoF of the Flower Basis Functions}

The number of degrees of freedom (DoF) of the loop basis functions can be found in [9]. This section discusses the DoF of the flower basis functions on a simply connected surface.

Assume that the surface is triangularly meshed with $N_{\text {node }}$ nodes, so the number of the associated flower basis functions is also $N_{\text {node }}$. For the sake of convenience, we define several terminologies as follows.

(1) Two nodes are called RWG-connected if they are the two free vertexes of a RWG base. We can draw a companion graph of the triangular mesh, which contains all nodes of the mesh structure but not the edges. Two nodes are connected with a line in the companion graph if they are RWG-connected.

(2) Two nodes are called connected in the graph if there exists at least one path between them.

(3) A node group is a subcollection of the nodes of the triangular mesh. If all nodes in a node group are connected and all nodes that are connected to the nodes in the group also belong to the group, then the node group is called RWG-connected. Note that no connection exists between two RWG-connected node groups.

With these definitions, it can be checked that a mesh structure on a simply-connected surface may have several RWG-connected node groups. Close examination shows that each RWG-connected node group should contain at least one node of every triangle in the mesh structure. This observation can be demonstrated by the following deduction: if the three vertexes of any triangle belong to a RWG-connected node group $G_{1}$, then it can be checked that all the vertexes of the neighboring triangles must also belong to the node group $G_{1}$. We can continue this deduction to conclude that all nodes on the simply-connected surface belong to node group $G_{1}$. On the other hand, if the three vertexes of any triangle do not belong to a RWG-connected node group $G_{1}$, then following 
the same deduction process, we can conclude that there is no node in the mesh structure that belongs to node group $G_{1}$. This observation means that there are at most 3 RWGconnected node groups on a simply-connected surface.

Since it is assumed that the reference directions of the flower bases $\mathbf{f}_{n}^{\text {Flower }}(\mathbf{r})$ are all pointing away from their reference nodes, when constructing the flower basis functions in a RWG-connected node group, each RWG basis function in it will be visited exactly twice in opposite direction, so the summation of all flower basis functions in the node group should be zero; that is,

$$
\sum_{i=1}^{N_{\mathrm{ng}}} \mathbf{f}_{n, i}^{\text {Flower }}(\mathbf{r})=0 .
$$

Here, $N_{\text {ng }}$ is the number of nodes in the group. Note that in a RWG-connected node group, (6) is not true anymore if one node is dropped away. Consequently, the number of the linearly independent flower basis function on the mesh structure is $N_{\text {node }}-N_{G}$, where $N_{G}$ is the number of RWGconnected node groups associated with the mesh structure and $1 \leq N_{G} \leq 3$.

Hereafter, a triangular mesh structure is called qualified for the flower basis functions if it contains only one RWGconnected node group. It can be proved that unqualified mesh structures are very seldom to encounter if not created deliberately. Some regular mesh structures can possibly have 2 or 3 RWG-connected node groups. Figure 2 shows such a mesh structure, which has 3 RWG-connected groups with their nodes labeled with different markers. This kind of mesh structure can also be found on closed surfaces.

Given a triangular mesh structure with $N_{\text {node }}$ nodes, it is easy to determine the number of RWG-connected node groups $N_{G}$ using the following searching routine:

(1) start from an arbitrarily-selected node, find all its RWG-connected vertexes, and mark them as new node elements in node group $G_{1}$;

(2) select, respectively, each new element in $G_{1}$ as the starting node and repeat step (1). Add all new nodes into node group $G_{1}$;

(3) repeat steps (2) till all nodes of group $G_{1}$ are found;

(4) the searching routine ends if the number of nodes in group $G_{1}$ equals $N_{\text {node }}$; otherwise, go to the next step;

(5) select a node that does not belong to group $G_{1}$, and go to step (1).

The searching routine ends when all nodes are assigned to a node group. Obviously, the searching routine can be terminated whenever a mesh triangle is found that all its three nodes belong to the group $G_{1}$, and in this case, there is exactly only one RWG-connected node group; hence, $N_{G}=1$.

More importantly, there exists a simple way to modify an unqualified triangular mesh structure to a qualified one as shown in Figure 3: first, select one triangle that has at least two neighboring triangles (e.g., the shadowed triangle); second, add two extra nodes in the two neighboring triangles, one node in one triangle (the two black-square nodes), and connect them with the corresponding vertexes (dotted lines). With these auxiliary meshes, it can be checked that the three nodes of the shadowed triangle are RWG-connected. Therefore, from the previously discussed observation, it can be verified that all nodes on the mesh structure are RWGconnected; thus, the modified mesh structure is qualified.

Consider the situation that the surface charge density is expressed with node-based basis functions. On a simplyconnected surface with $N_{\text {node }}$ nodes, the number of degrees of freedom of the surface charge density should be $N_{\text {node }}-1$ because the total charge on the surface should be zero [2] or remain constant. If the flower basis functions are used for the surface charge density, it is required that the number of the linearly independent flower basis functions should also be $N_{\text {node }}-1$; otherwise, the functional space spanned by the flower bases is not complete. Apparently, the above-defined qualified mesh structure meets this requirement. In order to generate a set of linearly independent flower bases, only one node should be dropped away in a qualified mesh structure.

On the other hand, (4) shows that the functional space spanned by the flower basis functions is actually a subspace of that spanned by the star basis functions. According to (4), a flower basis function is proportional to the average value of the star basis functions surrounding the reference node of the flower basis function. The error caused by this approximation will surely decrease with the refinement of the mesh structure. However, the numerical error due to this spatial averaging is indeed mesh-dependent. A general rule is to avoid mesh structures that too many edges are connected to a single node. An irregularly-defined flower basis function is shown in Figure 4. It contains 20 RWGs (or star bases), with an unusually large spatial span compared to other flower bases.

\section{Application of Loop-Flower Basis Functions}

The surface current can be expanded with loop-flower basis functions as

$$
\mathbf{J}_{s}=\sum_{n=1}^{N_{\text {Loop }}} j_{n}^{L_{1}} \mathbf{f}_{n}^{\text {Loop }}+\sum_{n=1}^{N_{\text {Hower }}} j_{n}^{F} \mathbf{f}_{n}^{\text {Flower }} .
$$

For qualified mesh structures consisting of $N_{\text {node }}$ nodes, $N_{\text {Flower }}=N_{\text {node }}-1$, while $N_{\text {Loop }}$ can be determined according to rules described in [9]. Following a standard discretization routine, the EFIE is transformed to a matrix system as follows:

$$
\left[\begin{array}{ll}
\mathbf{Z}^{\mathrm{FF}} & \mathbf{Z}^{\mathrm{FL}} \\
\mathbf{Z}^{\mathrm{LF}} & \mathbf{Z}^{\mathrm{LL}}
\end{array}\right]\left[\begin{array}{l}
\mathbf{J}^{F} \\
\mathbf{J}^{L}
\end{array}\right] \triangleq \mathbf{Z}\left[\begin{array}{l}
\mathbf{J}^{F} \\
\mathbf{J}^{L}
\end{array}\right]=\left[\begin{array}{l}
\mathbf{e}^{F} \\
\mathbf{e}^{L}
\end{array}\right]
$$

where the upper script " $L$ " stands for variables concerning with the loop basis functions and " $F$ " stands for the flower basis functions. $\mathrm{Z}^{\mathrm{FF}}, \mathrm{Z}^{\mathrm{LL}}, \mathrm{Z}^{\mathrm{LF}}, \mathrm{Z}^{\mathrm{FL}}$ are coupling matrices associated with the loop-flower basis functions, respectively. For example, the entries of $\mathbf{Z}^{\mathrm{LF}}$ are calculated with

$$
\mathrm{Z}^{\mathrm{LF}}(m, n)=\left\langle\mathbf{f}_{m}^{\mathrm{Loop}}, \mathscr{T}\left(\mathbf{f}_{n}^{\text {Flower }}\right)\right\rangle,
$$


where the EFIE operator $\mathscr{T}$ is defined by

$$
\begin{aligned}
\mathscr{T}\left(\mathbf{J}_{s}\right)= & -j \omega \mu \widehat{\mathbf{n}} \times \int_{S} g\left(\mathbf{r}, \mathbf{r}^{\prime}\right) \mathbf{J}_{s} d S^{\prime} \\
& +(j \omega \varepsilon)^{-1} \widehat{\mathbf{n}} \times \nabla \int_{S} g\left(\mathbf{r}, \mathbf{r}^{\prime}\right) \nabla_{t}^{\prime} \cdot \mathbf{J}_{s} d S^{\prime} .
\end{aligned}
$$

$g\left(\mathbf{r}, \mathbf{r}^{\prime}\right)$ is the scalar Green's function in the environment. $\mathbf{J}^{F}$, $\mathbf{J}^{L}$ are column vectors containing the expanding coefficients of the nonsolenoidal and the solenoidal currents, respectively. $\mathbf{e}^{F}, \mathbf{e}^{L}$ are the exciting vectors. Since $\nabla_{t}^{\prime} \cdot \mathbf{f}_{n}^{\text {Loop }} \equiv 0$, only the entries of $\mathbf{Z}^{\mathrm{FF}}(m, n)$ include the contribution from the second term in the right hand side of (10). At low frequencies, the matrix elements have different scaling properties with respect to frequency [3]. A balanced scaling scheme for (8) is

$$
\left[\begin{array}{cc}
k \mathbf{Z}^{\mathrm{FF}} & \mathbf{Z}^{\mathrm{FL}} \\
\mathbf{Z}^{\mathrm{LF}} & k^{-1} \mathbf{Z}^{\mathrm{LL}}
\end{array}\right]\left[\begin{array}{c}
k^{-1} \mathbf{J}^{F} \\
\mathbf{J}^{L}
\end{array}\right]=\left[\begin{array}{c}
\mathbf{e}^{F} \\
k^{-1} \mathbf{e}^{L}
\end{array}\right],
$$

where $k$ is the wave number. The scaling strategy in (11) is well-used in loop-star decomposition techniques and is also effective in loop-flower decomposition. The condition number of $\mathbf{Z}$ will be significantly reduced using (11) at very low frequencies.

The conventional RWG code can be used to get the coupling matrix by constructing transformation matrices from (1) and (3) as follows:

$$
\begin{aligned}
{\left[\mathbf{f}^{\text {Loop }}\right] } & =\mathbf{T}^{\text {Loop }} \cdot\left[\widetilde{\mathbf{f}}^{\text {RWG }}\right], \\
{\left[\mathbf{f}^{\text {Flower }}\right] } & =\mathbf{T}^{\text {Flower }} \cdot\left[\widetilde{\mathbf{f}}^{\text {RWG }}\right],
\end{aligned}
$$

where $\left[\mathbf{f}^{\text {Loop }}\right],\left[\tilde{\mathbf{f}}^{\text {RWG }}\right]$, and $\left[\mathbf{f}^{\text {Flower }}\right]$ are column vectors and $\mathbf{T}^{\text {Loop }}$ and $\mathbf{T}^{\text {Flower }}$ are transformation matrices. $\mathbf{T}^{\text {Loop }}$ is a sparse matrix with size of $\left(N_{\text {Loop }}, N_{\mathrm{RWG}}\right)$. At the $m$ th row, only several elements have nonzero values, which can be determined from (1). The transformation matrix $\mathbf{T}^{\text {Loop }}$ maps the $\tilde{\mathbf{f}}^{\mathrm{RWG}}$ bases to the $\mathbf{f}^{\text {Loop }}$ bases. Similarly, $\mathbf{T}^{\text {Flower }}$ maps the $\widetilde{\mathbf{f}}^{\text {RWG }}$ bases to the $\mathbf{f}^{\text {Flower }}$ bases. It is a sparse matrix of size $\left(N_{\text {Flower }}, N_{\text {RWG }}\right)$, whose elements can be determined from (3).

Define the coupling matrix associated with RWGs as

$$
\mathbf{Z}^{\mathrm{RWG}}(m, n)=\left\langle\widetilde{\mathbf{f}}_{m}^{\mathrm{RWG}}, \mathscr{T}\left(\widetilde{\mathbf{f}}_{n}^{\mathrm{RWG}}\right)\right\rangle
$$

Then it can be derived that

$$
\mathbf{Z}^{u v}=\mathbf{T}^{u} \cdot \mathbf{Z}^{\mathrm{RWG}} \cdot\left(\mathbf{T}^{v}\right)^{t},
$$

where the upper script " $u, v$ " can be either "Loop" or "Flower," and " $t$ " means transpose.

\section{Calderon Preconditioner Based on Loop-Flower Basis Functions}

Since the ill-conditioning of the coefficient matrix $\mathbf{Z}$ is rooted in the spectral properties of the EFIE operator $\mathscr{T}$, it cannot be improved unless the spectral of EFIE operator is modified.
Calderon Identity [6] is often used to make the operator $\mathscr{T}$ more regular as follows:

$$
\mathscr{T}^{2}=\mathscr{K}^{2}+\frac{\mathscr{I}}{4}
$$

where the operator $\mathscr{K}$ is compact on a smooth surface [15] and is defined by

$$
\mathscr{K}\left(\mathbf{J}_{s}\right)=\widehat{\mathbf{n}} \times \int_{S} \nabla g\left(\mathbf{r}, \mathbf{r}^{\prime}\right) \times \mathbf{J}_{s} d S^{\prime}
$$

Applying $\mathscr{T}$ to EFIE yields

$$
\mathscr{T}\left\{\mathscr{T}\left\{\mathbf{J}_{s}\right\}\right\}=\mathscr{T}\left\{-\widehat{\mathbf{n}} \times \mathbf{E}^{\text {in }}(\mathbf{r})\right\} .
$$

According to the Calderon Identity, the discretized system of (17) is expected to be well-conditioned. Assume that the left and right operator are discretized with $\mathbf{f}_{n}^{\text {Loop }}, \mathbf{f}_{n}^{\text {Flower }}$ (for sources) and $\widehat{\mathbf{n}} \times \mathbf{f}_{n}^{\text {Loop }}, \widehat{\mathbf{n}} \times \mathbf{f}_{n}^{\text {Flower }}$ (for testing). The corresponding Gram matrix $\mathbf{P}$ can be written in block matrices as follows:

$$
\mathbf{P}=\left[\begin{array}{ll}
\mathbf{P}^{\mathrm{FF}} & \mathbf{P}^{\mathrm{FL}} \\
\mathbf{P}^{\mathrm{LF}} & \mathbf{P}^{\mathrm{LL}}
\end{array}\right]
$$

the entries of which are the inner products of the testing and expansion bases. For example, the entries of $\mathbf{P}^{L F}$ read

$$
\mathbf{P}^{\mathrm{LF}}(m, n)=\left\langle\widehat{\mathbf{n}} \times \mathbf{f}_{m}^{\mathrm{Loop}}(\mathbf{r}), \mathbf{f}_{n}^{\text {Flower }}(\mathbf{r})\right\rangle .
$$

On a closed surface, both the number of degrees of freedom of the loop basis functions and that of the flower basis functions should be $N_{\text {node }}-1$; hence, $\mathbf{P}^{\mathrm{LF}}$ is a square matrix. We will show that $\mathbf{P}^{\mathrm{LF}}$ is invertible if the triangular mesh structure is qualified for the flower basis functions.

The entries of the Gram matrix can be evaluated analytically. Assume that two modified RWGs $\widetilde{\mathbf{f}}_{p}^{\mathrm{RWG}}$ and $\widetilde{\mathbf{f}}_{q}^{\mathrm{RWG}}$ overlap on a triangle; the basic integration involved is found to be

$$
\operatorname{Int}_{p q}=\int_{S_{p}} \widehat{\mathbf{n}} \times \tilde{\mathbf{f}}_{p}^{\mathrm{RWG}} \cdot \widetilde{\mathbf{f}}_{q}^{\mathrm{RWG}} d S^{\prime}= \begin{cases} \pm \frac{1}{6}, & \mathbf{r}_{q} \neq \mathbf{r}_{p}, \\ 0, & \mathbf{r}_{q}=\mathbf{r}_{p} .\end{cases}
$$

Obviously, $\mathbf{P}^{\mathrm{LL}}(m, m)=0$ because $\mathbf{f}_{m}^{\mathrm{Loop}}(\mathbf{r})$ and $\widehat{\mathbf{n}} \times \mathbf{f}_{m}^{\mathrm{Loop}}(\mathbf{r})$ are orthogonal. If $m \neq n$, then $\mathbf{f}_{m}^{\text {Loop }}(\mathbf{r})$ and $\mathbf{f}_{n}^{\text {Loop }}(\mathbf{r})$ either share a RWG base (a pair of triangles) or have no overlapping area at all. In the former case, the integral (20) on the two triangles will exactly cancel each other. Consequently, it can be concluded that $\mathbf{P}^{\mathrm{LL}}(m, n)=0$ for all $m, n$. This induction is also applicable for $\mathbf{P}^{\mathrm{FF}}$. However, in the case of flower bases, it is possible that the overlapping area consists of RWGs and single triangles. The integral of (20) on the RWG triangle pairs can be cancelled, but that on the single triangles cannot be cancelled.

With the reference directions specified in Section 2, it is not difficult to check that $\mathbf{P}^{\mathrm{LF}}(m, m)=N_{m} / 3$, where $N_{m}$ is the number of triangles connecting to the node $\mathbf{r}_{m}$. It can also be 
verified that on the $m$ th row and the $m$ th column of $\mathbf{P}^{\mathrm{LF}}$, there exist at most $N_{m}$ off-diagonal nonzero elements and that

$$
\begin{gathered}
\left|\mathbf{P}^{\mathrm{LF}}(m, m)\right| \geq \sum_{n \neq m}\left|\mathbf{P}^{\mathrm{LF}}(m, n)\right|, \\
\left|\mathbf{P}^{\mathrm{LF}}(m, m)\right| \geq \sum_{n \neq m}\left|\mathbf{P}^{\mathrm{LF}}(n, m)\right| .
\end{gathered}
$$

Because one node has been dropped away in constructing the loop-flower bases, (21) includes strict inequality for at least one $m$. Consequently, $\mathbf{P}^{\mathrm{LF}}$ is a diagonal dominant matrix. It can be further proved that if the mesh structure contains only one RWG-connected node group, $\mathbf{P}^{\mathrm{LF}}$ is irreducible because its associated directed graph is strongly connected. Mathematically, irreducible diagonal dominant matrix is invertible. On the other hand, we can check that $\mathbf{P}^{\mathrm{LF}}$ is a symmetrical matrix and $\mathbf{P}^{\mathrm{FL}}=-\mathbf{P}^{\mathrm{LF}}$. Therefore, the two nondiagonal submatrices in the Gram matrix $\mathbf{P}$ are both invertible, while $\mathbf{P}^{\mathrm{LL}}$ is a zero matrix and $\mathbf{P}^{\mathrm{FF}}$ is bounded. It can be readily concluded from these observations that the Gram matrix $\mathbf{P}$ expressed in (18) is invertible. Although, the proof has not been extended to open surfaces, the Gram matrices in numerical examples of open surfaces that we have checked are all invertible.

Denote $\widetilde{\mathbf{e}}^{L, F}(m)=\left\langle-\mathbf{E}^{\text {in }}(\mathbf{r}), \mathbf{f}_{m}^{\text {Loop, Flower }}\right\rangle$; then, the Calderon preconditioned EFIE can be written in matrix form as follows:

$$
\mathbf{Z P}^{-1} \mathbf{Z} \cdot \mathbf{J}=\mathbf{Z} \mathbf{P}^{-1} \cdot \widetilde{\mathbf{e}}_{i n}
$$

where $\mathbf{J}$ is the column vector of the surface current and $\mathbf{A}=$ $\left(\mathbf{Z} \cdot \mathbf{P}^{-\mathbf{1}}\right)$ is the preconditioning matrix. Note that in $(22)$, the two matrices associated with the left and right operator are the same, while in BC-CPs, the two matrices are different.

\section{Numerical Examples}

Four PEC scatterers are analyzed: a sphere, a cube, an almond, and a square plate. In all these examples, the incident waves are $x$-polarized plane waves with amplitude of $1 \mathrm{~V} / \mathrm{m}$, traveling along $+z$ axis. The polar and azimuthal angles are denoted by $\theta$ and $\phi$, respectively.

6.1. PEC Sphere. The radius of the sphere is $1 \mathrm{~m}$. The surface mesh structure has totally 290 nodes, 576 triangles, and 864 edges, with an average edge length of $0.225 \mathrm{~m}$. As a result, there are 864 RWGs, 289 loop basis functions, 289 flower basis functions, and 575 star basis functions. The scattering problem is analyzed at 11 discrete frequency sampling points, from $0.01 \mathrm{~Hz}$ to $100 \mathrm{MHz}$.

The biRCSs at $\phi=0^{\circ}$ (denoted by $\mathrm{RCS}_{\theta \theta}$ ) are calculated with EFIEs. The RCSs at $0.01 \mathrm{~Hz}$ are plotted in Figure 5. It can be seen that the biRCSs calculated by using loop-star bases (LS), loop-flower bases (LF), and the loop-flower bases in conjunction with Calderon preconditioner (LF-CP) all agree well with the analytical results obtained by using Mie series expansion. However, the results obtained by using RWGs are not correct due to the effect of low frequency breakdown.

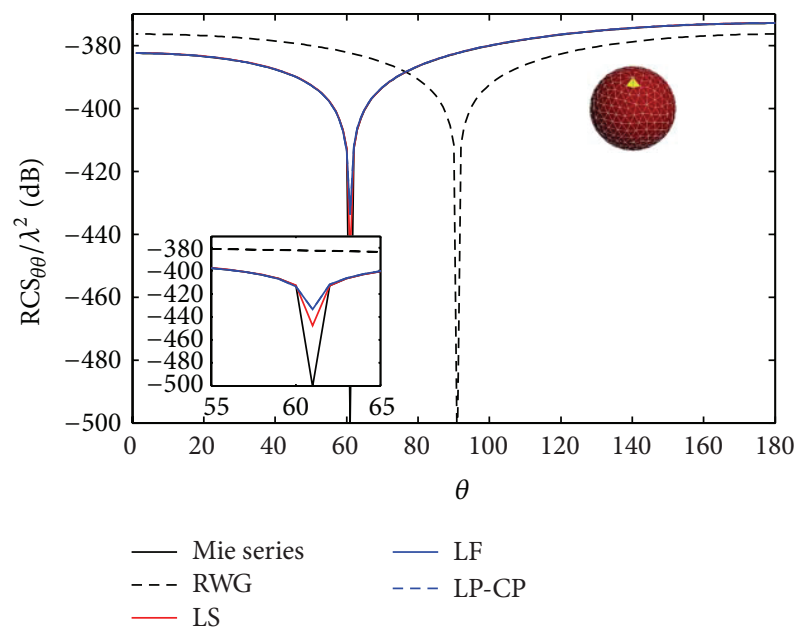

Figure 5: BiRCS $\left(\phi=0^{\circ}\right)$ of the PEC sphere @ $0.01 \mathrm{~Hz}$.

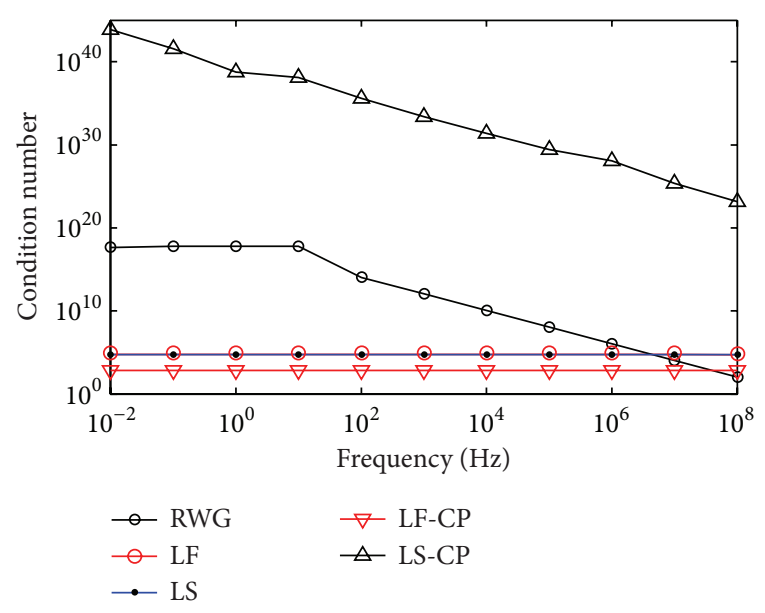

FigURE 6: Condition numbers versus frequency.

The condition numbers of the coefficient matrices are plotted in Figure 6. It is obvious that using loop-flower decomposition technique can avoid low frequency breakdown, just like using loop-star decomposition. The condition numbers associated with LF and LS remain almost unchanged at very low frequencies while EFIE-RWG fails. The condition numbers of the coefficient matrices are further decreased by about 100 times in this example when the Calderon preconditioner, realized directly using loop-flower basis functions (LF-CP), is added. For comparison, those condition numbers corresponding to the RWG-based loopstar bases in conjunction with Calderon preconditioner (LS$\mathrm{CP}$ ) are also plotted in Figure 6, which confirms the fact that Calderon preconditioners for EFIEs cannot be implemented using RWG-based loop-star basis functions alone.

As has been verified with this example, the loopflower basis function can be directly used for implementing Calderon preconditioners that are valid even at very low frequencies. The two Calderon preconditioners, that is, LF$\mathrm{CP}$ and $\mathrm{BC}-\mathrm{CP}$, are compared with this example, and a slightly coarse surface mesh is used, which has 74 nodes 


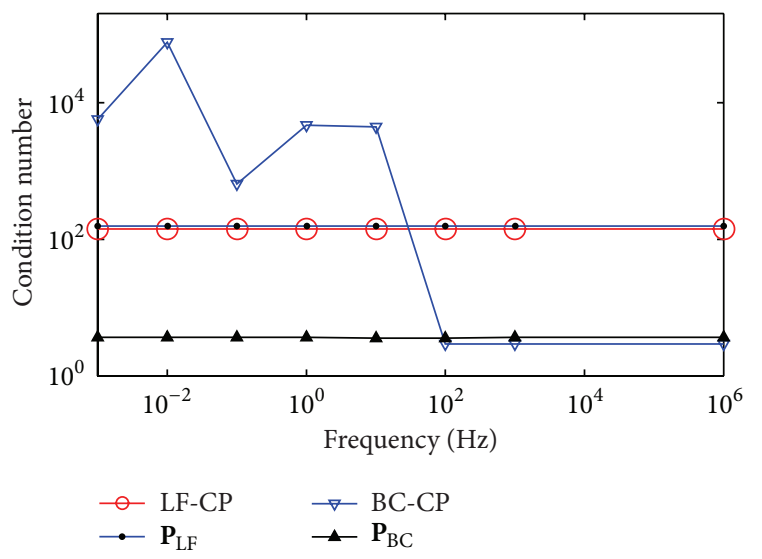

FIgURE 7: The condition numbers of the coupling matrices and the corresponding Gram matrices.

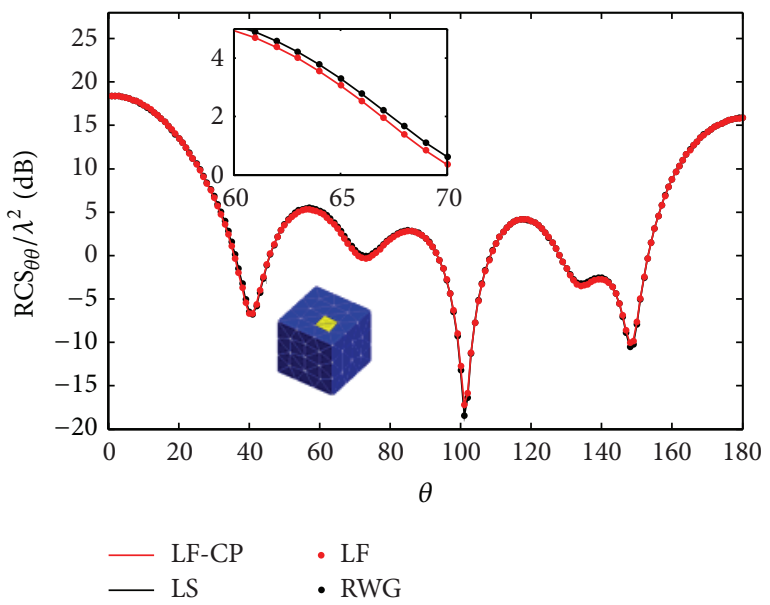

Figure 8: BiRCSs of the PEC cube @ 0.5 GHz.

and 216 RWGs. In order to implement BC-CP, $(216 \times 6=$ 1296) barycentric RWGs have to be created. The sizes of the coupling matrix and the Gram matrix associated with the barycentric RWGs are all $(1296 \times 1296)$, much larger than that associated with the original RWGs. However, the condition number of the coupling matrix after applying BC$\mathrm{CP}$, if it does not fail, is much smaller than that after applying LF-CP. Figure 7 shows that LF-CP remains stable at very low frequencies but BC-CP may fail if no techniques, such as dividing the operator to a smooth part and a hyper-singular part, are used.

The condition numbers of the associated Gram matrices are also checked. In Figure 7, $\mathbf{P}_{\mathrm{LF}}$ and $\mathbf{P}_{\mathrm{BC}}$, respectively, represent the Gram matrix associated with LF-CP and BC-CP. It can be seen that the condition numbers of the coefficient matrices are approximately equal to that of the associated Gram matrices.

6.2. PEC Cube. The PEC cube checked here has an edge length of $1 \mathrm{~m}$. There are totally 440 nodes, 876 triangles, and 1314 edges in the mesh structure, resulting in 1314 RWGs, 439 flower and loop bases, and 875 star basis functions. The

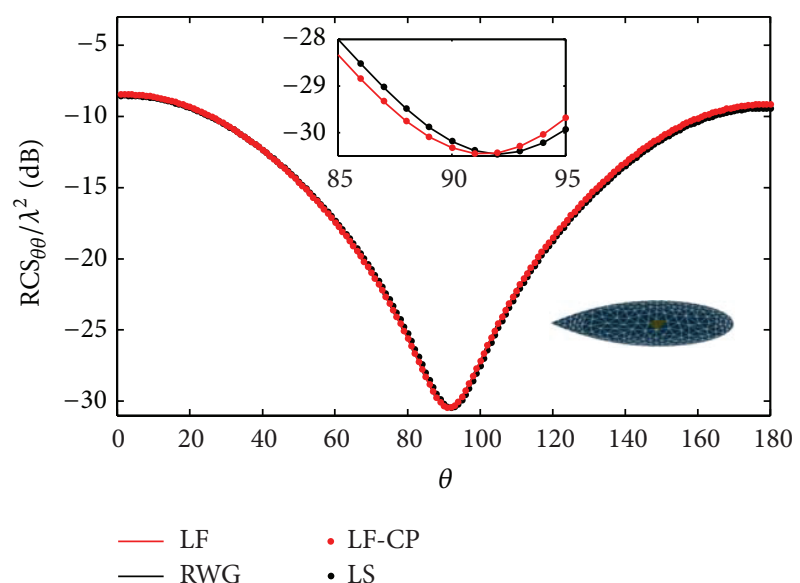

(a)

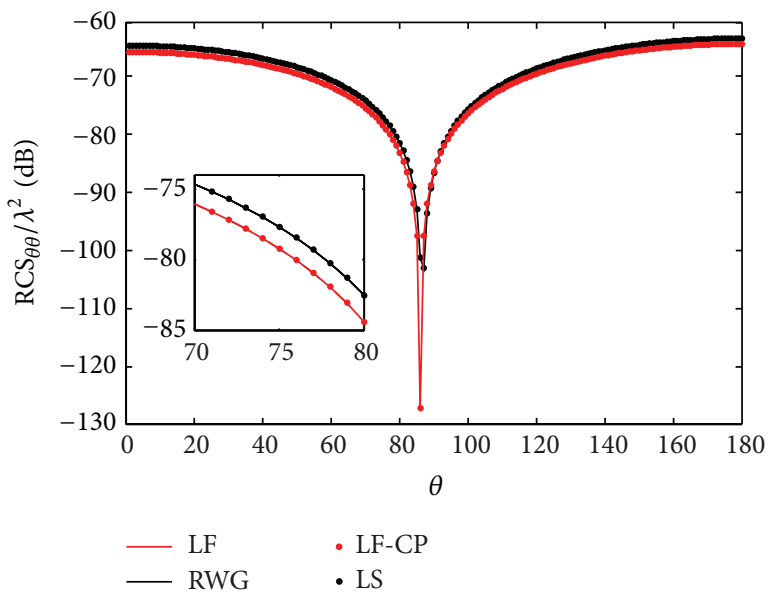

(b)

FIGURE 9: BiRCSs of the PEC almond. (a) $1 \mathrm{GHz}$ and (b) $70 \mathrm{MHz}$.

working frequency is assumed to be $0.5 \mathrm{GHz}$, and the average length is about $0.2 \lambda$. Since it is not a low frequency problem, (8) is used in this example. The calculated RCSs are plotted in Figure 8 . They agree well with each other.

6.3. PEC Almond. The PEC almond has maximum dimensions of $(0.25 \mathrm{~m}, 0.1 \mathrm{~m}$, and $0.032 \mathrm{~m})$. The surface mesh contains 292 nodes, 580 triangles, and 870 edges, resulting in 870 RWGs, 291 linearly independent loop-flower bases, and 579 star bases. The average edge length is $0.01 \mathrm{~m}$ and the minimum edge length is $0.0035 \mathrm{~m}$. The numerical results at $1 \mathrm{GHz}$ and $70 \mathrm{MHz}$ are shown in Figure 9. In this case, there is a small discrepancy between the results of LF and LS. As has been pointed out previously, the functional space spanned by the flower basis functions is a subspace of that spanned by star basis functions. At the sharp edges of the PEC almond, the flower basis functions are not accurate enough to catch the surface current property with the given mesh size.

6.4. PEC Plate. A square PEC plate with size of $1 \mathrm{~m} \times 1 \mathrm{~m}$ is considered. Its mesh structure is shown in Figure 10, with 121 


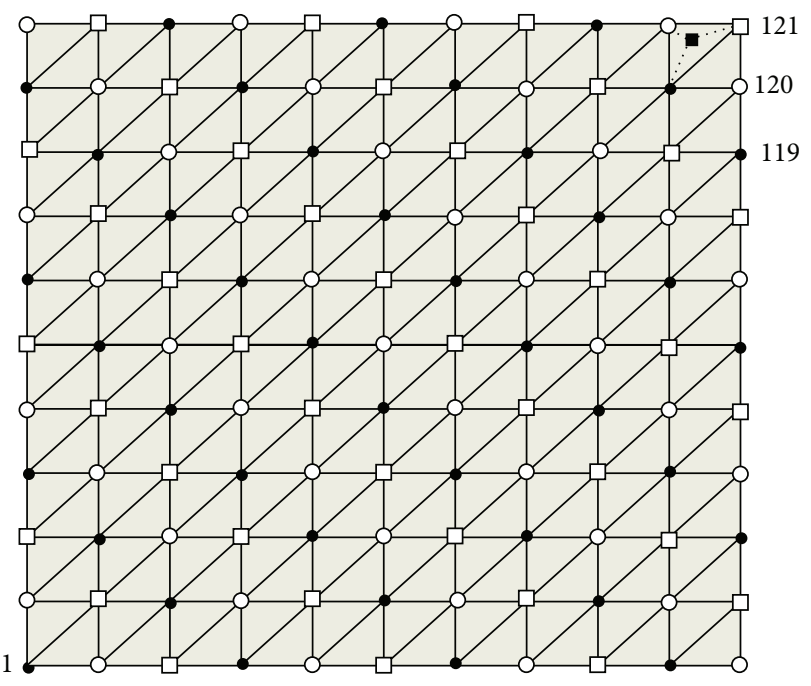

FIgURE 10: PEC plate mesh structure.

nodes (including 81 internal nodes), 280 RWGs, resulting in 80 loop basis functions. Using the searching routine, 3 RWG node groups are created. Their nodes are marked with black dots, empty circles, and empty squares, respectively, as shown in Figure 10. Therefore, one node in each node group should be dropped away, and the number of linearly independent flower basis functions is 118. In this example, the three nodes labeled by 119, 120, and 121 are dropped away (note that their coefficients can be determined from the others). The working frequency is assumed to be $70 \mathrm{MHz}$. The currents obtained by using loop-flower basis functions and loop-flower bases in conjunction with Calderon preconditioner agree well with that obtained using RWG bases, as shown in Figure 11, where the expansion coefficients of the RWG basis functions are plotted. The corresponding $\mathrm{RCS}_{\theta \theta}$ are shown in Figure 12.

Although a set of linearly independent flower basis functions can be obtained by dropping away three nodes, the numerical errors in surface current may become slightly larger near these three nodes. A better way is to transform the mesh structure to a qualified one using the auxiliary meshes shown in Figure 3. In this example, it can be checked that the mesh structure becomes qualified for flower basis functions by adding only one node and three edges in a triangle (the black-square and dotted edges in Figure 10). The numerical results are not presented here because they agree very well with those using RWGs.

\section{Conclusions}

It has been shown with numerical examples that the loopflower basis functions are effective for analyzing electromagnetic scattering problems on closed surfaces as well as open surfaces. The loop flower basis functions can also be used to implement Calderon preconditioners for EFIEs directly. Key issues need to be further investigated, such as the approximation properties of the loop-flower bases, the condition number of the associated Gram matrix, and the application to dielectric scatterers.

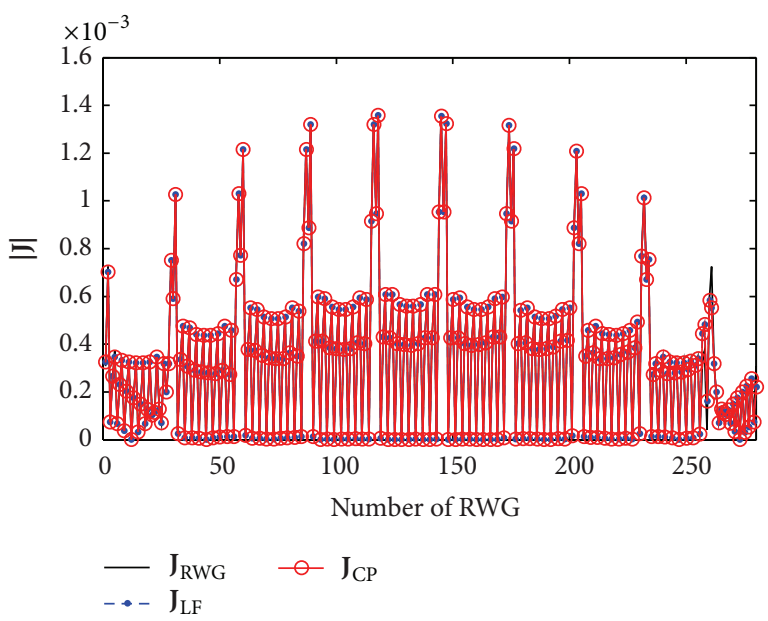

FIGURE 11: Surface currents on the PEC plate (expansion coefficients of the RWG basis functions).

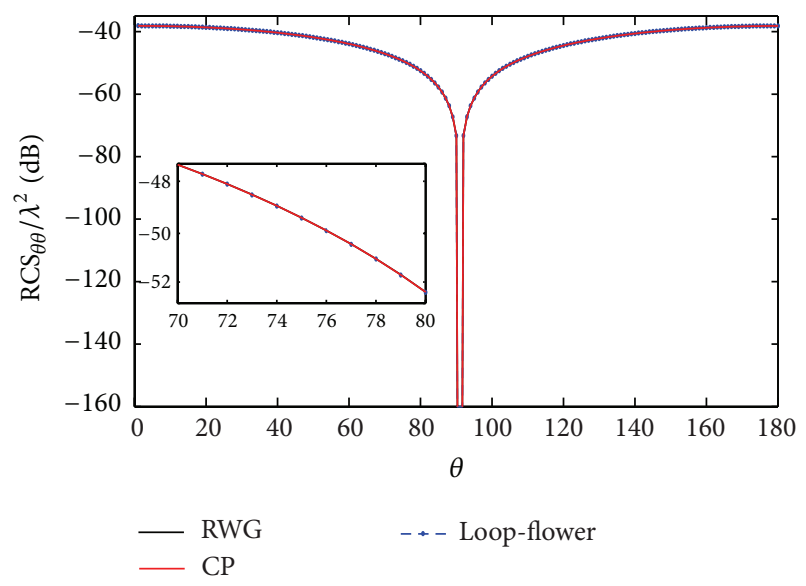

Figure 12: BiRCSs of the PEC plate.

\section{Conflict of Interests}

The authors declare that there is no conflict of interests regarding the publication of this paper.

\section{Acknowledgment}

This work is supported by the SAST foundation 2013.

\section{References}

[1] S. M. Rao, D. R. Wilton, and A. W. Glisson, "Electromagnetic scattering by surfaces of arbitrary shape," IEEE Transactions on Antennas and Propagation, vol. 30, no. 3, pp. 409-418, 1982.

[2] G. Vecchi, "Loop-star decomposition of basis functions in the discretization of the EFIE," IEEE Transactions on Antennas and Propagation, vol. 47, no. 2, pp. 339-346, 1999.

[3] J.-S. Zhao and W. C. Chew, "Integral equation solution of Maxwell's equations from zero frequency to microwave frequencies," IEEE Transactions on Antennas and Propagation, vol. 48, no. 10, pp. 1635-1645, 2000. 
[4] J. F. Lee, R. Lee, and R. J. Burkholder, "Loop star basis functions and a robust preconditioner for EFIE scattering problems," IEEE Transactions on Antennas and Propagation, vol. 51, no. 8, pp. 1855-1863, 2003.

[5] T. F. Eibert, "Iterative-solver convergence for loop-star and loop-tree decompositions in method-of-moments solutions of the electric-field integral equation," IEEE Antennas and Propagation Magazine, vol. 46, no. 3, pp. 80-85, 2004.

[6] G. C. Hsiao and R. E. Kleinman, "Mathematical foundations for error estimation in numerical solutions of integral equations in electromagnetics," IEEE Transactions on Antennas and Propagation, vol. 45, no. 3, pp. 316-328, 1997.

[7] A. Buffa and S. H. Christiansen, "A dual finite element complex on the barycentric refinement," Mathematics of Computation, vol. 76, no. 260, pp. 1743-1769, 2007.

[8] F. P. Andriulli, K. Cools, H. Bağci et al., "A multiplicative Calderon preconditioner for the electric field integral equation," IEEE Transactions on Antennas and Propagation, vol. 56, no. 8, pp. 2398-2412, 2008.

[9] M. B. Stephanson and J. F. Lee, "Preconditioned electric field integral equation using Calderon identities and dual loop/star basis functions," IEEE Transactions on Antennas and Propagation, vol. 57, no. 4, pp. 1274-1279, 2009.

[10] S. Yan, J. M. Jin, and Z. Nie, "EFIE analysis of low-frequency problems with loop-star decomposition and Calderón multiplicative preconditioner," IEEE Transactions on Antennas and Propagation, vol. 58, no. 3, pp. 857-867, 2010.

[11] R. D. Graglia, D. R. Wilton, and A. F. Peterson, "Higher order interpolatory vector bases for computational electromagnetics," IEEE Transactions on Antennas and Propagation, vol. 45, no. 3, pp. 329-342, 1997.

[12] R. D. Graglia, A. Peterson, and L. Matekovits, "Singular, hierarchical scalar basis functions for triangular cells," IEEE Transactions on Antennas and Propagation, vol. 61, no. 7, pp. 3674-3692, 2013.

[13] R. D. Graglia, A. F. Peterson, L. Matekovits, and P. Petrini, "Issues associated with additive basis functions for the finite element treatment of corner singularities," Electromagnetics, vol. 33, no. 3-4, 2014.

[14] R. D. Graglia, A. Peterson, L. Matekovits, and P. Petrini, "Singular hierarchical curl-conforming vector bases for triangular cells," IEEE Transactions on Antennas and Propagation. In press.

[15] J. C. Nedelec, Acoustic and Electromagnetic Equations, Springer, New York, NY, USA, 2000. 

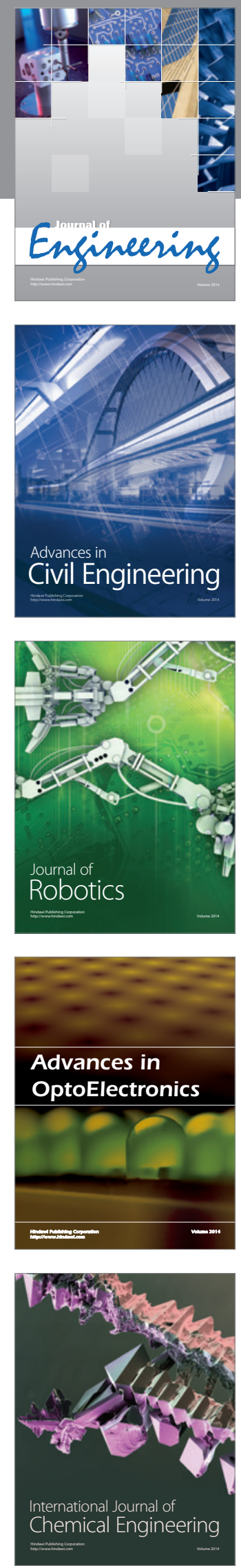

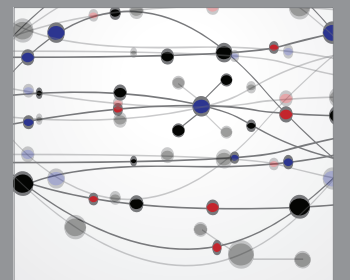

The Scientific World Journal
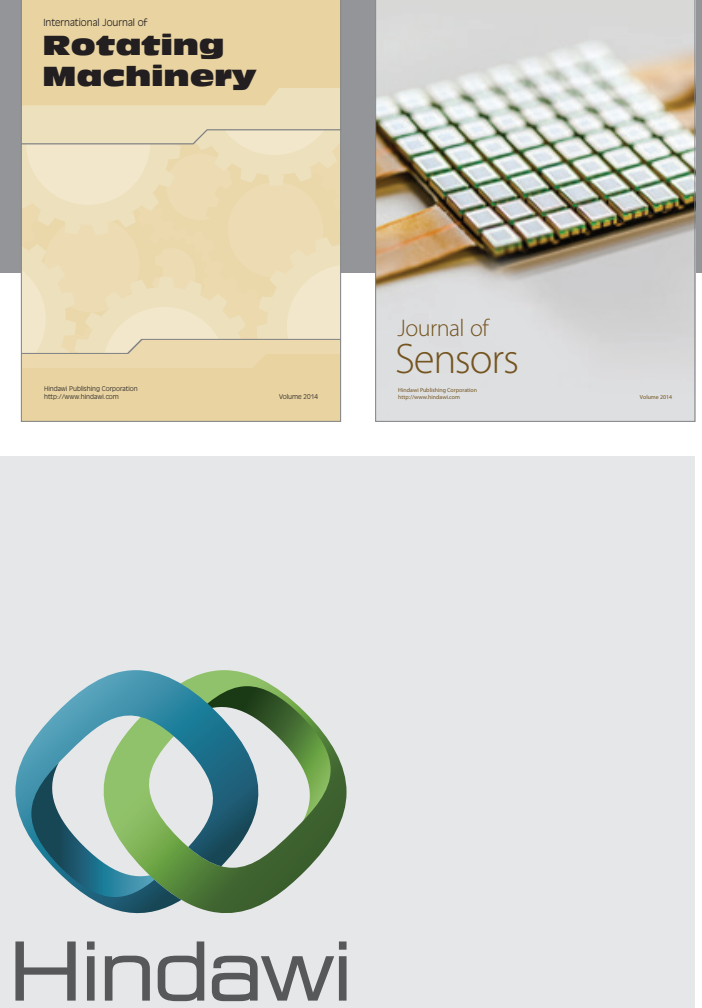

Submit your manuscripts at http://www.hindawi.com
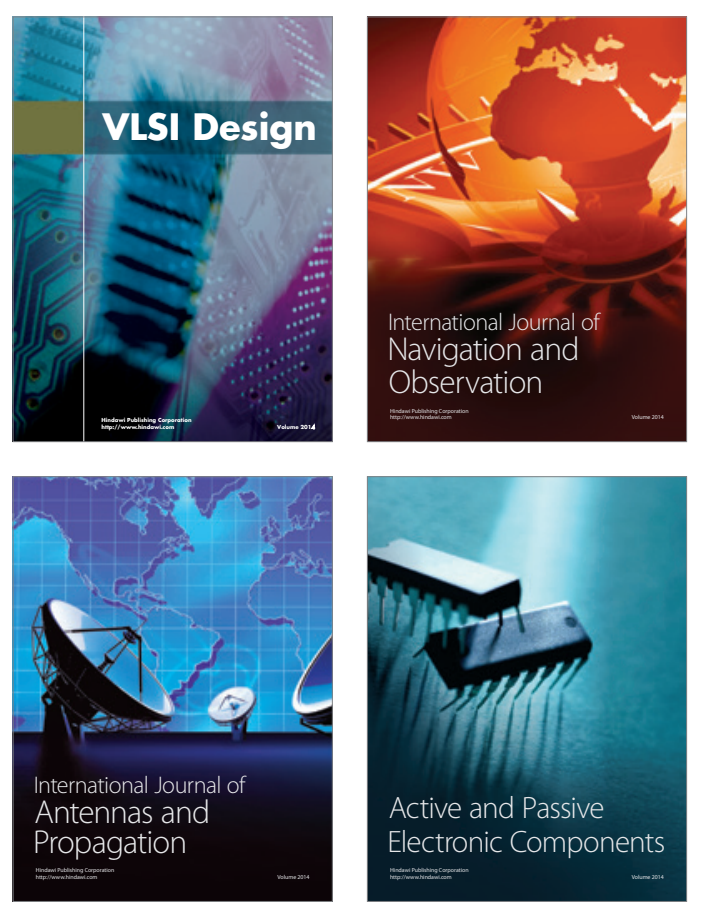
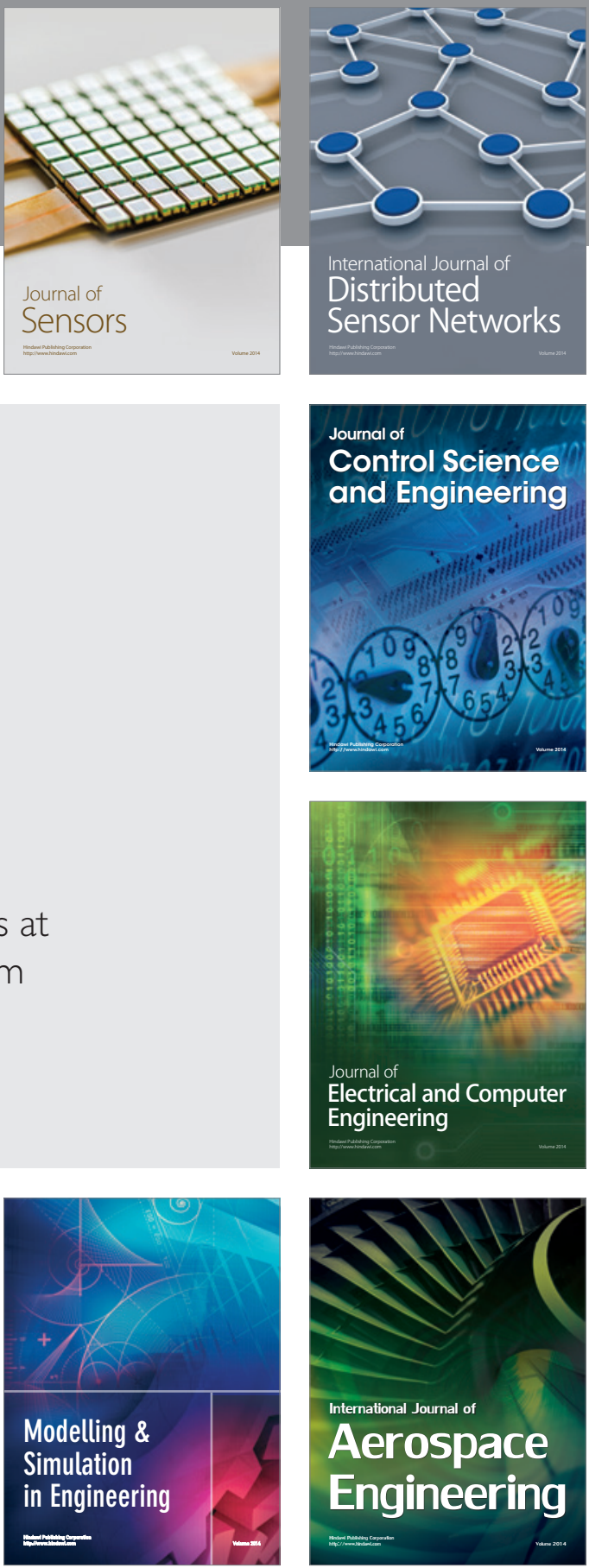

Journal of

Control Science

and Engineering
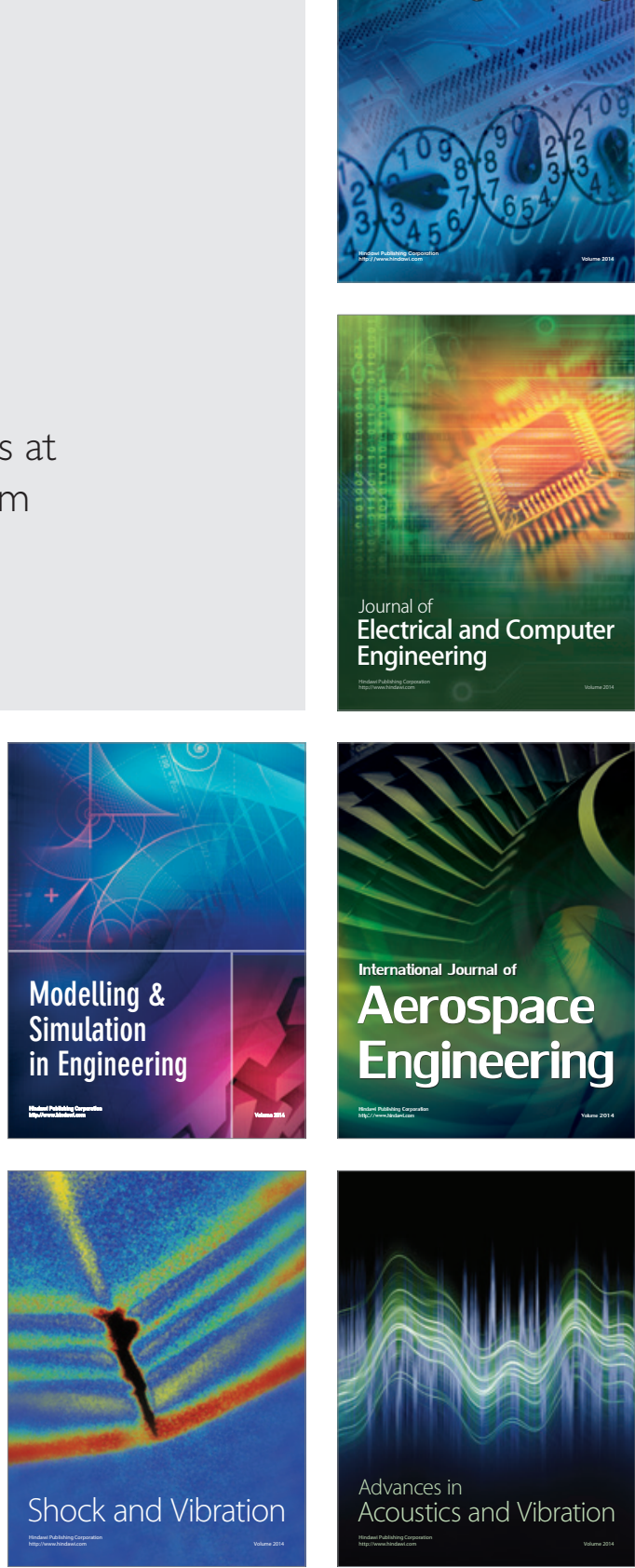